\title{
Direct versus Bridging Mechanical Thrombectomy in Elderly Patients with Acute Large Vessel Occlusion: A Multicenter Cohort Study
}

\author{
Yating Jian,' Lili Zhao,' \\ Baixue Jia, ${ }^{2} \mathrm{Xu}$ Tong, ${ }^{2}$ \\ Tao Li, ' Yulun Wu,' \\ Xiaoya Wang, 'Zhen Gao,' \\ Yu Gong,' Xuelei Zhang, ${ }^{2}$ \\ Huqing Wang, ' Ru Zhang, \\ Lei Zhang, ' \\ Zhongrong Miao, ${ }^{2}$ \\ Guilian Zhang' \\ On behalf of ANGEL-ACT \\ Study Group \\ 'Department of Neurology, The Second \\ Affiliated Hospital of Xi'an Jiaotong \\ University, Xi'an, 7I0004, Shaanxi, People's \\ Republic of China; ${ }^{2}$ Department of \\ Interventional Neuroradiology, Beijing \\ Tiantan Hospital, Capital Medical \\ University, Beijing, People's Republic of \\ China
}

Correspondence: Guilian Zhang Department of Neurology, the Second Affiliated Hospital of Xi'an Jiaotong University, No. 157 Xiwulu, Xi'an, 7I0004, Shaanxi, People's Republic of China

Tel +862987679346

Fax +86 2987678599

Email zhgl_2006@I26.com

Zhongrong Miao

Department of Interventional

Neuroradiology, Beijing Tiantan Hospital,

Capital Medical University, No. 119 South

West Road, Fengtai District, Beijing,

People's Republic of China

Tel +86I360I243293

Email zhongrongm@163.com
Purpose: Elderly people represent a growing stroke population with different pathophysiological states than younger. Whether intravenous thrombolysis (IVT) before mechanical thrombectomy (MT) is beneficial for elderly patients remains unclear. This study compared the efficacy and safety between elderly patients treated with MT alone and those treated with both IVT and MT.

Patients and Methods: Patients aged $\geq 65$ years who were eligible for IVT within $4.5 \mathrm{~h}$ from symptom onset were selected from the ANGEL-ACT (Endovascular Treatment Key Technique and Emergency Work Flow Improvement of Acute Ischemic Stroke) registry, a prospective registry program for patients with endovascular treatment from 111 Chinese stroke centers. The primary efficacy outcome was the 90-day modified Rankin Scale score. We compared efficacy and safety outcomes using ordinal or binary logistic regression or a generalized linear model.

Results: In total, 482 elderly patients were included: 187 (38.8\%) received IVT and MT (bridging MT) and 295 (61.2\%) received MT alone (direct MT). There was no significant difference in the 90day modified Rankin Scale score between the two groups (median: 4 vs 4 points, respectively; adjusted $\beta=-0.048, P=0.822$ ). The direct MT group had a shorter onset-to-puncture time (225 vs $255 \mathrm{~min}$, respectively; adjusted $\beta=-55.074, P=0.002)$ and a lower rate of parenchymal hemorrhage type 2 within $24 \mathrm{~h}(2.80 \%$ vs $6.63 \%$, respectively; adjusted odds ratio [OR] $=0.287,95 \%$ confidence interval $[\mathrm{CI}]=0.096-0.856, P=0.025)$. In addition, the direct MT group showed a trend toward a lower incidence of $\operatorname{sICH}(5.67 \%$ vs $10.06 \%$, adjusted $\mathrm{OR}=0.453, P=0.061)$, procedure-related complications $(7.12 \%$ vs $12.30 \%$, adjusted $\mathrm{OR}=0.499, P=0.052)$ and distal or new territorial embolization ( $4.07 \%$ vs $6.95 \%$, adjusted $\mathrm{OR}=0.450, P=0.093$ ).

Conclusion: Direct MT had similar efficacy to bridging MT in terms of the 90-day functional outcome in elderly patients, whereas bridging MT had a longer onset-to-puncture time and increased risk of hemorrhagic transformation and procedure-related complications.

Keywords: acute ischemic stroke, endovascular treatment, intravenous thrombolysis, elderly patients

\section{Introduction}

Mechanical thrombectomy (MT) is now the standard treatment for patients with acute ischemic stroke (AIS) caused by large vessel occlusion (LVO). Current guidelines recommend that patients eligible for intravenous thrombolysis (IVT) should receive IVT even if MT is being considered. ${ }^{1}$ However, there is some evidence that combining IVT with MT (bridging MT) may result in similar outcomes to MT alone (direct MT), including from observational studies, ${ }^{2-5}$ systematic reviews and meta-analyses, ${ }^{6,7}$ and two recently published randomized controlled trials (the DIRECT-MT ${ }^{8}$ and the DEVT ${ }^{9}$ ). 
The progressive increase in life expectancy and aging of the population has resulted in a growing number of elderly patients with stroke. However, elderly patients have different pathophysiological states and more comorbidities than younger patients. Although the American Heart Association/American Stroke Association (AHA/ ASA) guidelines show that IV alteplase treatment is recommended for patients aged $>80$ years under certain conditions, ${ }^{1}$ elderly patients have a higher risk of complications, including a higher risk of hemorrhagic transformation (HT) after IVT. ${ }^{10}$ Nevertheless, the efficacy of pretreatment with IVT prior to MT in elderly patients and whether this procedure increases the risk of HT remains unclear. Thus, the efficacy and safety of bridging and direct MT in elderly patients requires further study. Therefore, based on the data of the ANGEL-ACT (Endovascular Treatment Key Technique and Emergency Work Flow Improvement of Acute Ischemic Stroke; ClinicalTrial.gov ID: NCT03370939), a prospective, nationwide, multicenter registry in China, we explored the efficacy and safety of direct MT and bridging MT in elderly patients.

\section{Patients and Methods Study Population and Treatment}

Our study population included patients registered in the ANGEL-ACT from November 2017 to March 2019. The ANGEL-ACT is a nationwide, multicenter, prospective registry program for patients who undergo endovascular treatment for acute LVO from 111 stroke centers in China. ${ }^{11}$ This research program was approved by the ethics committee of the Second Affiliated Hospital of Xi'an Jiaotong University and each participating site. Each participant or his/her representative gave written informed consent before being enrolled in the study. This study was conducted in accordance with the Declaration of Helsinki.

The inclusion criteria were (1) the time from onset-tohospital admission $<4.5 \mathrm{~h}$; (2) computed tomography angiography, magnetic resonance angiography, or digital subtraction angiography confirmation of LVO, defined as occlusion of the internal carotid artery, vertebral artery, basilar artery, middle cerebral artery (M1/M2), anterior cerebral artery (A1/A2), and posterior cerebral artery (P1); (3) age of $\geq 65$ years; and (4) treatment with MT. Patients with contraindications for IVT and missing primary efficacy outcomes were excluded. In total, 482 eligible patients were included in this study. The patients were divided into the bridging MT group (IVT+MT) and the direct MT group (MT without IVT) according to whether they received IVT.

Each patient or his/her legally authorized representative was fully informed of the purpose, cost and complications of IVT and MT. After full consideration, some patients chose to undergo direct MT even if they were transferred from the primary hospital to an advanced stroke center. The main reasons for not performing IVT included refusal by patients or their family members, fear of the side effect of IVT by physicians, and for the purpose of reducing financial burden. ${ }^{11}$ Each participant or his/her legally authorized representative provided written informed consent before IVT and MT treatment. IVT was administered according to the current AHA/ASA guidelines within $4.5 \mathrm{~h}$ from symptom onset in the majority of patients (alteplase $0.9 \mathrm{mg} / \mathrm{kg}$, maximum $90 \mathrm{mg}$, over 1 $\mathrm{h}$ with $10 \%$ initial bolus ${ }^{1}$ ). The interventional neurologists of all centers had received standard training and were highly experienced in interventional neuroradiology. Stent retriever, aspiration, or a combination of both methods were included. The type and size of the equipment to be used in the operation, as well as other rescue therapies such as balloon angioplasty, stent implantation, or arterial thrombolysis, were determined based on the interventional neurologists' experience.

\section{Data Collection}

Demographic data, vascular risk factors, imaging and laboratory data, stroke and surgery details, and 90-day functional outcomes were prospectively collected. The National Institutes of Health Stroke Scale (NIHSS) score and the modified Rankin Scale (mRS) score, which represent the severity of stroke and the recovery of neurological function, respectively, were recorded by trained and qualified professional investigators. The NIHSS scores were recorded at baseline and at $24 \mathrm{~h}$ and $7 \mathrm{~d}$ after admission. Images were evaluated by an independent laboratory of 10 trained neuroradiologists who were blinded to the clinical data and outcomes. Two neuroradiologists independently evaluated each image; when their results were inconsistent, the final results were decided upon by a third physician.

\section{Outcomes}

Outcomes were divided into efficacy and safety outcomes. The primary efficacy outcome was the mRS score at 90 
days, with an excellent functional outcome being defined as an mRS score of 0 or 1 , a good functional outcome as $\mathrm{mRS}$ score of 0 to 2 , and a favorable clinical outcome as mRS score of 0 to 3 . The secondary efficacy outcomes were changes in the NIHSS score from baseline to 24 $\mathrm{h}$ and 7 days, successful recanalization, door-to-groin puncture time, onset-to-groin puncture time, puncture-torecanalization time, number of thrombectomy passes, and total number of operations. Successful recanalization was defined as a modified Thrombolysis in Cerebral Infarction score of $\geq 2 \mathrm{~b}$. The puncture-to-recanalization time was defined as the duration of time from the moment of puncture to the first successful recanalization or the last contrast bolus (when the vessel was not successfully recanalized). The total number of operations was defined as the sum of all endovascular treatments including stent retriever, aspiration, balloon angioplasty, and stent implantation.

The main safety outcomes were symptomatic intracranial hemorrhage (sICH) within $24 \mathrm{~h}$ as defined by the criteria of the Heidelberg Bleeding Classification. ${ }^{12}$ The secondary safety outcomes were (1) any ICH within $24 \mathrm{~h}$; (2) parenchymal hemorrhage type 2 ( $\mathrm{PH}-2)$ within 24 $\mathrm{h}$ defined as blood clots in $>30 \%$ of the infarcted area with a substantial space occupying effect; ${ }^{13}$ (3) mortality within 90 days; and (4) procedure-related complications, including distal or new territorial embolization, vascular perforation, dissection, and vasospasm.

\section{Statistical Analysis}

All continuous variables are presented as median with interquartile range (IQR), while categorical variables are presented as frequency and percentage. Differences in continuous variables were analyzed with the MannWhitney $U$-test, while categorical variables were analyzed with the $\chi 2$ test or Fisher's exact test. The 90 -day mRS score was treated as an ordinal and dichotomous variable and was analyzed with ordinal and binary logistic regression, respectively. Other binary outcomes were analyzed with logistic regression, while continuous outcomes were analyzed using a generalized linear model. A multivariate model was run to adjust for the use of IVT and variables with a $P$-value of $<0.05$ in the baseline characteristics. A second model was run to add the baseline NIHSS score, Alberta Stroke Program Early Computed Tomography Score (ASPECTS), location of the occluded vessels, and etiology as additional covariates. All statistical analyses were performed using statistical software
(SAS v9.4; SAS Institute Inc, Cary, NC, USA). A $P$-value of $<0.05$ was considered statistically significant.

\section{Results}

A total of 1793 patients were included in the ANGELACT registry. 690 patients met the inclusion criteria, of whom 180 were ineligible for IVT and 28 were missing the main efficacy outcome. Finally, 482 patients with AIS treated with MT were included in the present study; 187 (38.8\%) patients received IVT and MT (bridging MT) and $295(61.2 \%)$ patients received MT alone (direct MT). A patient flowchart is shown in Figure 1.

The median patient age was 73 years (IQR, 69-78 years), and the median baseline NIHSS score was 17 (IQR, 13-22). Of the 482 enrolled patients, 259 $(53.73 \%)$ were men and $392(81.33 \%)$ had anterior circulation stroke. A total of $426(88.38 \%)$ patients underwent stent thrombectomy, while $86(17.84 \%)$ underwent aspiration. The median onset-to-door time was $90 \mathrm{~min}$ (IQR, 50$140 \mathrm{~min}$ ). Most patients were directly transported to an endovascular-capable center, while 131 (27.18\%) were transferred from the primary hospital to an advanced stroke center.

\section{Baseline Characteristics}

The baseline characteristics of patients who underwent direct MT and bridging MT are shown in Table 1. There was a higher proportion of men (58.31\% vs $46.52 \%$, respectively; $P=0.012$ ), use of preoperative anticoagulants (5.42\% vs $0.53 \%$, respectively; $P=0.004)$, and use of intraoperative heparin $(57.97 \%$ vs $32.09 \%$, respectively; $P<0.001)$ in the direct MT group than in the bridging MT group, while the proportion of patients undergoing aspiration catheterization was higher in the bridging MT group than in the direct MT group $(25.13 \%$ vs $13.22 \%$, respectively; $P=0.001)$. There were no significant differences in any other factors between the two groups $(P>0.05)$.

\section{Efficacy Outcomes}

The median mRS at 90 days was 4 both in the direct MT group and the bridging MT group, and there was no significant difference between the two groups $(P=0.858$; Table 2). The distribution of $\mathrm{mRS}$ scores of $0-6$ at 90 days is shown in Figure 2. There were no significant differences in the proportions of an excellent clinical outcome (35.83\% vs $34.24 \%$, respectively; $P=0.721$; Table 2 ), good functional outcome $(37.97 \%$ vs $38.31 \%$, 


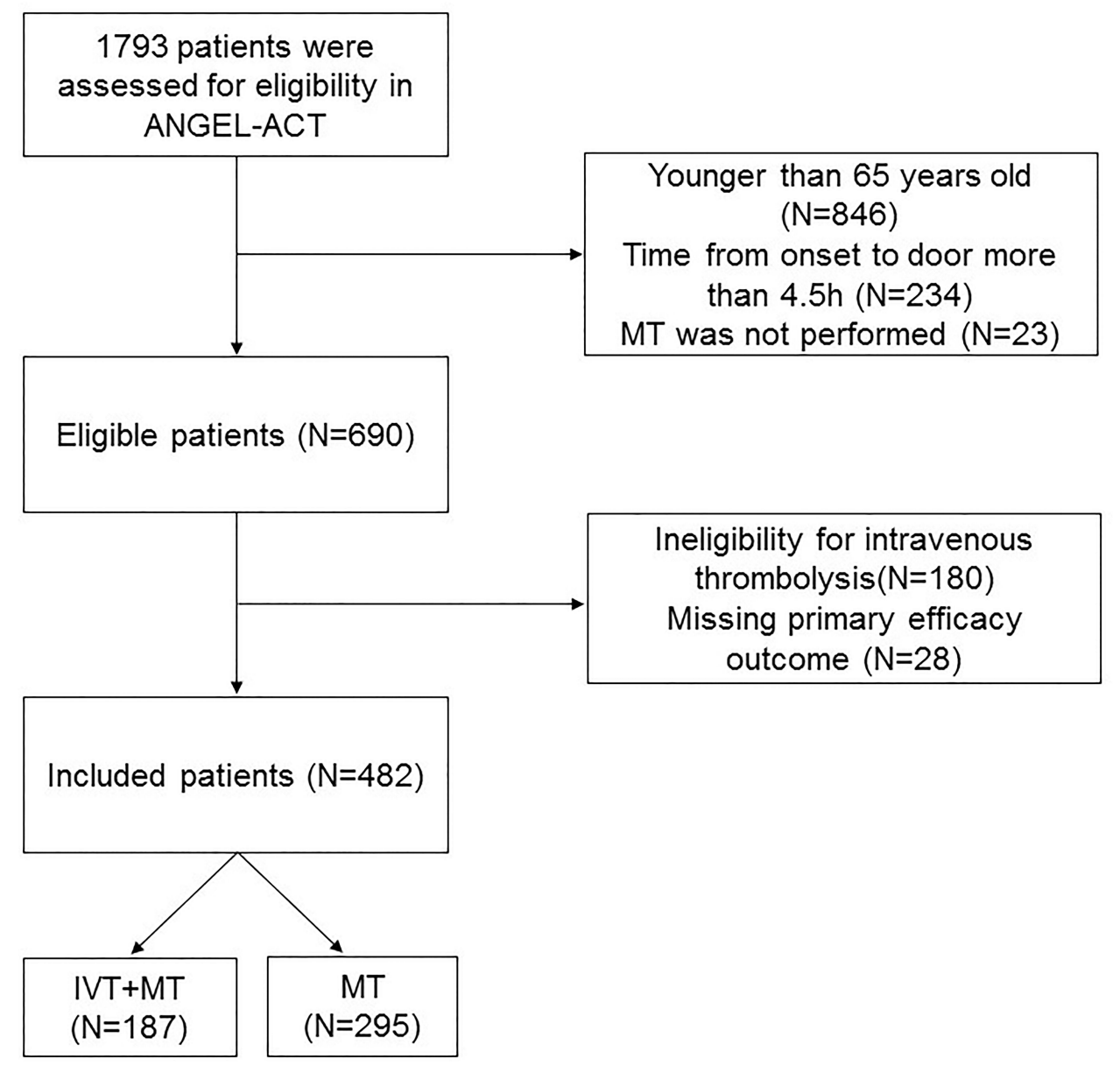

Figure I Flow chart of the patient inclusion steps.

respectively; $P=0.941$; Table 2 ), or favorable clinical outcome ( $45.99 \%$ vs $48.81 \%$, respectively; $P=0.546$; Table 2 ) between the two groups. Ordinal logistic regression and binary logistic regression based on the classification of 0 $1,0-2$, and 0-3 showed no significant difference in the 90day $\mathrm{mRS}$ score $(P>0.05$ for each; Table 3$)$. In total, 88 $(18.26 \%)$ patients were $\geq 80$ years old. A subgroup analysis stratified by age (65-79 vs $\geq 80$ years) showed no significant difference in the ratio of a 90-day mRS score of $0-1$, 0-2, and 0-3 (Table 4).

The direct MT group had a shorter onset-to-puncture time than the bridging MT group (225 vs $255 \mathrm{~min}$, respectively; $P<0.001$; Table 2). This finding remained unchanged after controlling for other factors by multiple linear regression (model 1: adjusted $\beta=-59.94518$, $P=0.001$; model 2: adjusted $\beta=-55.07449, \quad P=0.002$; Table 3). Although there were significant differences in the 24-h NIHSS score change ( $P=0.045$; Table 2$)$, number of thrombectomy passes $(P=0.009$; Table 2$)$, and total number of operations ( $P=0.009$; Table 2$)$, the multivariate analysis showed no significant differences after adjusting for other factors $(P>0.05$ for each; Table 3$)$.

\section{Safety Outcomes}

Univariate analysis showed a trend toward a lower incidence of PH-2 within $24 \mathrm{~h}$ in the direct MT group than in the bridging MT group (2.80\% vs $6.63 \%$, respectively; $P=0.053$; Table 2). Further, after adjusting for other factors, multivariate logistic regression analysis confirmed that the incidence of PH-2 was lower in the direct MT group (model 1: $\mathrm{OR}=0.318, \quad 95 \% \quad \mathrm{CI}=0.114-0.890$, $P=0.029 ;$ model 2: $\mathrm{OR}=0.287,95 \% \mathrm{CI}=0.096-0.856$, $P=0.025$; Table 3 ). The direct $\mathrm{MT}$ group also showed a trend toward a lower incidence of $\mathrm{sICH}(5.67 \%$ vs $10.06 \%$, respectively; model 1 : $\mathrm{OR}=0.522, P=0.093$; model 2: $\mathrm{OR}=0.453, P=0.061$; Table 3), procedurerelated complications $(7.12 \%$ vs $12.30 \%$, respectively; model 1: $\mathrm{OR}=0.463, P=0.027$; model 2: $\mathrm{OR}=0.499$, $P=0.052$; Table 3 ) and distal or new territorial embolizations $(4.07 \%$ vs $6.95 \%$, respectively; model $1: \mathrm{OR}=0.403$, 
Table I Comparison of Baseline Characteristics Between Direct Mechanical Thrombectomy (MT) and Bridging MT Patients

\begin{tabular}{|c|c|c|c|c|}
\hline Characteristics & Total $(n=482)$ & BMT $(n=187)$ & DMT $(n=295)$ & $P$-value \\
\hline Age, median (IQR), year & $73(69-78)$ & $72(68-78)$ & $73(69-78)$ & 0.791 \\
\hline Male (n, \%) & $259(53.73)$ & $87(46.52)$ & $172(58.3 \mid)$ & 0.012 \\
\hline \multicolumn{5}{|l|}{ Medical history $(\mathrm{n}, \%)$} \\
\hline Hypertension & $309(64.11)$ & $115(61.50)$ & $194(65.76)$ & 0.342 \\
\hline Diabetes mellitus & $97(20.12)$ & $36(19.25)$ & $6 \mathrm{I}(20.68)$ & 0.703 \\
\hline Dyslipidemia & $34(7.05)$ & $18(9.63)$ & $16(5.42)$ & 0.079 \\
\hline Coronary heart disease & $108(22.4 \mathrm{I})$ & $46(24.60)$ & $62(21.02)$ & 0.358 \\
\hline Atrial fibrillation & $221(45.85)$ & $85(45.45)$ & $136(46.10)$ & 0.890 \\
\hline Prior stroke & $106(21.99)$ & $33(17.65)$ & $73(24.75)$ & 0.067 \\
\hline Current smoking & $348 / 391(89.00)$ & I38/I55 (89.03) & $210 / 236(88.98)$ & 0.988 \\
\hline SBP, median (IQR), mmHg & $147(|33-16|)$ & $146(133-165)$ & $148(132-160)$ & 0.980 \\
\hline NIHSS score, median (IQR) & $17(13-22)$ & $17(|3-2|)$ & $18(13-23)$ & 0.084 \\
\hline Serum glucose, median (IQR) & $7.0(5.9-9.2)$ & $7.1(6.0-9.1)$ & $7.0(5.8-9.3)$ & 0.864 \\
\hline WBC count, median (IQR) & $7.7(6.3-9.8)$ & $7.5(6.4-9.9)$ & $7.8(6.3-9.7)$ & 0.932 \\
\hline Platelet count, median (IQR) & $190(152-232)$ & $191(156-232)$ & $190(152-232)$ & 0.525 \\
\hline INR, median (IQR) & $1.02(0.96-1.10)$ & $1.01(0.95-1.10)$ & $1.03(0.97-1.10)$ & 0.224 \\
\hline Serum creatinine, median (IQR) & $71.0(60.4-83.3)$ & $72.0(60.0-84.5)$ & $71.0(60.6-83.0)$ & 0.996 \\
\hline ASPECTS, median (IQR) & $10(7-10)$ & $10(7-10)$ & $10(7-10)$ & 0.213 \\
\hline \multicolumn{5}{|l|}{ Occlusion site (n, \%) } \\
\hline Anterior circulation & $392(81.33)$ & I58 (84.49) & $234(79.32)$ & 0.156 \\
\hline Posterior circulation & $90(18.67)$ & $29(15.5 I)$ & $61(20.68)$ & \\
\hline Good collaterals (n, \%) & $109 / 206(52.91)$ & $37 / 75(49.33)$ & $72 / 131$ (54.96) & 0.436 \\
\hline \multicolumn{5}{|l|}{ TOAST classification (n, \%) } \\
\hline Large artery atherosclerosis & $198 / 480(41.25)$ & $75 / 186(40.32)$ & I23/294 (4I.84) & 0.551 \\
\hline Cardioembolism & $217 / 480(45.21)$ & $89 / 186(47.85)$ & I28/294 (43.54) & \\
\hline Other or unknown etiology & $65 / 480(13.54)$ & $22 / 186(11.83)$ & $43 / 294(14.63)$ & \\
\hline \multicolumn{5}{|l|}{ Treatment $(\mathrm{n}, \%)$} \\
\hline Preoperative antiplatelet agents & $81(16.80)$ & $26(13.90)$ & $55(18.64)$ & 0.175 \\
\hline Preoperative anticoagulants & $17(3.53)$ & I $(0.53)$ & $16(5.42)$ & $0.004 *$ \\
\hline General anesthesia & $184(38.17)$ & $74(39.57)$ & II 0 (37.29) & 0.615 \\
\hline Stent retriever & $426(88.38)$ & $162(86.63)$ & $264(89.49)$ & 0.340 \\
\hline Aspiration catheter & $86(17.84)$ & $47(25.13)$ & $39(13.22)$ & 0.001 \\
\hline Intra-arterial thrombolysis & $44(9.13)$ & 14 (7.49) & $30(10.17)$ & 0.319 \\
\hline Balloon angioplasty & 79 (16.39) & $35(18.72)$ & $44(14.92)$ & 0.272 \\
\hline Stenting & $63(13.07)$ & $25(13.37)$ & $38(12.88)$ & 0.877 \\
\hline Intraoperative heparin & $231(47.93)$ & $60(32.09)$ & I7I (57.97) & $<0.001$ \\
\hline Infusion of GP Ilb/Illa inhibitor & $205(42.53)$ & $74(39.57)$ & $|3|(44.4 \mid)$ & 0.296 \\
\hline OTD time, median (IQR), min & $90(50-140)$ & $90(53-140)$ & $90(48-144)$ & 0.583 \\
\hline Drip and ship (n, \%) & $131(27.18)$ & $5 \mathrm{I}(27.27)$ & $80(27.12)$ & 0.970 \\
\hline
\end{tabular}

Notes: Good collaterals: American Society of Interventional and Therapeutic Neuroradiology/Society of Interventional Radiology $\geq 2$; *Represents Fisher's exact test. Abbreviations: DMT, direct mechanical thrombectomy; BMT, bridging mechanical thrombectomy; SBP, systolic blood pressure; NIHSS, National Institutes of Health Stroke Scale; WBC, white blood cell count; INR, international normalized ratio; ASPECTS, Alberta Stroke Program Early CT Score; OTD, symptom onset to door. 
Table 2 Univariate Analysis of Outcomes in the Direct Mechanical Thrombectomy (MT) and Bridging MT Patients

\begin{tabular}{|c|c|c|c|c|}
\hline Outcomes & BMT & DMT & OR/ß $(95 \% \mathrm{Cl})$ & $P$-value \\
\hline \multicolumn{5}{|l|}{ Efficacy outcome } \\
\hline $\mathrm{mRS}$ at $90 \mathrm{~d}$, median (IQR) & $4(1-5)$ & $4(1-5)$ & 0.03738 & 0.858 \\
\hline $\mathrm{mRS} 0-1$ at $90 \mathrm{~d}$ & $67(35.83)$ & I0I (34.24) & $0.932(0.635-1.368)$ & 0.721 \\
\hline $\mathrm{mRS} 0-2$ at $90 \mathrm{~d}$ & 7I (37.97) & $\mathrm{II} 3(38.3 \mathrm{I})$ & $1.014(0.696-1.479)$ & 0.941 \\
\hline $\mathrm{mRS} 0-3$ at $90 \mathrm{~d}$ & $86(45.99)$ & I 44 (48.8I) & $1.120(0.776-1.617)$ & 0.546 \\
\hline $\begin{array}{l}\text { Change in NIHSS score at } 24 \mathrm{~h} \text {, } \\
\text { median (IQR) }\end{array}$ & $-2(-6,0)$ & $-2(-7,0)$ & -1.56836 & 0.045 \\
\hline $\begin{array}{l}\text { Change in NIHSS score at } 7 \mathrm{~d} \text {, } \\
\text { median (IQR) }\end{array}$ & $-9(-14,-3)$ & $-9(-14,-4)$ & -1.12063 & 0.275 \\
\hline Successful recanalization & $163(87.17)$ & $258(87.46)$ & I.027 (0.592-I.779) & 0.925 \\
\hline OTP time, median (IQR), min & $255(199-322)$ & $225(175-273)$ & -61.53800 & $<0.001$ \\
\hline DTP, median (IQR), min & 144 (100-207) & $109(76-158)$ & -45.91377 & 0.060 \\
\hline PTR time, median (IQR), min & $87(53-123)$ & $83(50-123)$ & 0.99663 & 0.857 \\
\hline Number of MT passes, median (IQR) & $2(1-3)$ & $I(I-2)$ & -0.36955 & 0.009 \\
\hline $\begin{array}{l}\text { Total number of operation, } \\
\text { median (IQR) }\end{array}$ & $2(I-3)$ & $2(I-3)$ & -0.36790 & 0.009 \\
\hline \multicolumn{5}{|l|}{ Safety outcomes } \\
\hline Symptomatic ICH within $24 \mathrm{~h}$ & $18 / 179(10.06)$ & $16 / 282(5.67)$ & $0.538(0.267-1.085)$ & 0.083 \\
\hline Any $\mathrm{ICH}$ within $24 \mathrm{~h}$ & $51 / 181(28.18)$ & $62 / 286(21.68)$ & $0.706(0.459-1.083)$ & 0.111 \\
\hline $\mathrm{PH}-2$ within $24 \mathrm{~h}$ & $12 / 18 \mid(6.63)$ & $8 / 286(2.80)$ & $0.405(0.162-1.012)$ & 0.053 \\
\hline Death within $90 \mathrm{~d}$ & $37 / 143(25.87)$ & $54 / 237(22.78)$ & $0.845(0.522-1.369)$ & 0.494 \\
\hline Distal or new territorial embolization & $13(6.95)$ & $12(4.07)$ & $0.568(0.253-1.272)$ & 0.169 \\
\hline Arterial perforation & $5(2.67)$ & $8(2.7 I)$ & $1.015(0.327-3.149)$ & 0.980 \\
\hline Arterial dissection & $2(1.07)$ & $0(0)$ & - & 0.957 \\
\hline Arterial vasospasm & $4(2.14)$ & I $(0.34)$ & $0.156(0.017-1.403)$ & 0.097 \\
\hline Total complications & $23(12.30)$ & $21(7.12)$ & $0.546(0.293-1.018)$ & 0.057 \\
\hline
\end{tabular}

Abbreviations: OTP time, time from symptom onset to puncture; DTP time, time from door to puncture; PTR time, time from puncture to recanalization.

$P=0.047$; model 2: $\mathrm{OR}=0.450, P=0.093$; Table 3). There were no differences in other safety outcomes, including any ICH within $24 \mathrm{~h}$ and death within 90 days, between the two groups.

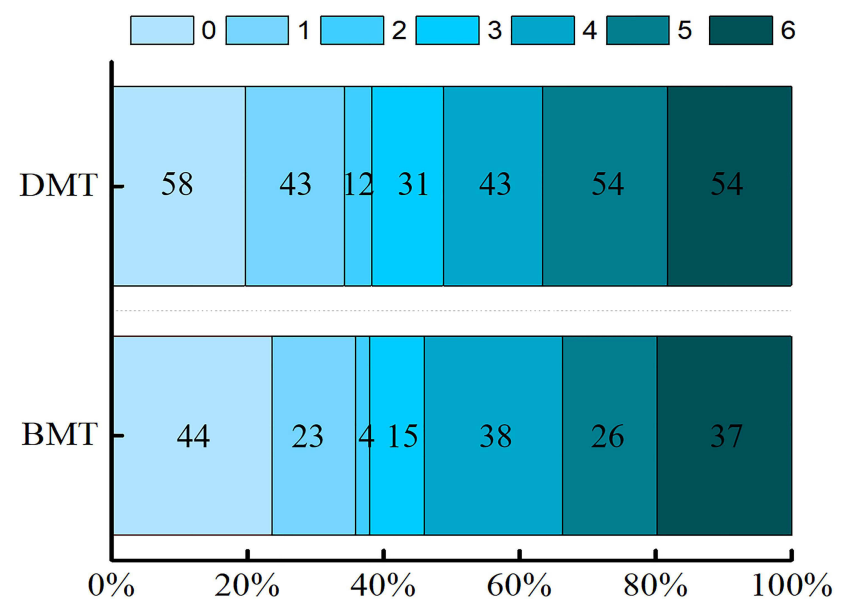

Figure 2 Distribution of the modified Rankin Scale (mRS) scores at 90 days. Abbreviations: BMT, bridging mechanical thrombectomy; DMT, direct mechanical thrombectomy.

\section{Discussion}

As a prospective registry study of 111 centers, the present study reflects the real-world situation of endovascular treatment for AIS in China. By comparing the efficacy and safety of MT with or without pre-treatment IVT in elderly patients, we found that direct MT had a similar 90day $\mathrm{mRS}$ score to that for bridging MT.

Two recently published randomized controlled trials (the DIRECT-MT trial ${ }^{8}$ and the DEVT trial ${ }^{9}$ ) proved that endovascular thrombectomy alone was noninferior to bridging MT in terms of functional outcome. Later, the SKIP trial ${ }^{14}$ showed that endovascular treatment alone and bridging MT had similarly favorable outcomes, although the trial failed to demonstrate noninferiority. Our findings are consistent with those trials. The patients in the above trial were $>18$ years old, and only the SKIP trial performed a subgroup analysis for ages of $<70$ and $\geq 70$ years; however, it showed no significant heterogeneity of effect on the mRS score. Previous data on the effects of direct MT and bridging MT in elderly patients predominantly comes from observational studies. For example, in 
Table 3 Multivariate Analysis of Outcomes in Elderly Patients

\begin{tabular}{|c|c|c|c|c|}
\hline & \multicolumn{2}{|c|}{ Model I } & \multicolumn{2}{|c|}{ Model 2} \\
\hline & $\begin{array}{l}\text { Adjusted OR/ } \beta \\
\qquad(95 \% \mathrm{Cl})\end{array}$ & $P$-value & $\begin{array}{l}\text { Adjusted OR/ } \beta \\
\qquad(95 \% \mathrm{Cl})\end{array}$ & $P$-value \\
\hline Efficacy outcome & & & & \\
\hline mRS at $90 \mathrm{~d}$, median (IQR) & 0.02080 & 0.925 & -0.04827 & 0.822 \\
\hline $\mathrm{mRS} 0-\mathrm{I}$ at $90 \mathrm{~d}$ & $0.945(0.629-1.418)$ & 0.784 & $0.976(0.634-1.503)$ & 0.914 \\
\hline $\mathrm{mRS} 0-2$ at $90 \mathrm{~d}$ & $1.003(0.672-1.496)$ & 0.990 & $1.050(0.687-1.604)$ & 0.822 \\
\hline $\mathrm{mRS} 0-3$ at $90 \mathrm{~d}$ & I.I85 (0.802-I.752) & 0.394 & $1.278(0.846-1.930)$ & 0.244 \\
\hline $\begin{array}{l}\text { Change in NIHSS score at } 24 \mathrm{~h} \text {, } \\
\text { median (IQR) }\end{array}$ & -1.81529 & 0.029 & -1.41694 & 0.075 \\
\hline $\begin{array}{l}\text { Change in NIHSS score at } 7 \mathrm{~d} \text {, } \\
\text { median (IQR) }\end{array}$ & -1.51778 & 0.165 & -0.74295 & 0.436 \\
\hline Successful recanalization & I.I58 (0.649-2.066) & 0.620 & I.I58 (0.64I-2.089) & 0.627 \\
\hline OTP time, median (IQR), min & -59.94518 & 0.001 & -55.07449 & 0.002 \\
\hline DTP time, median (IQR), min & -49.80116 & 0.053 & -44.82975 & 0.085 \\
\hline PTR time, median (IQR), min & 2.78556 & 0.634 & 2.97991 & 0.612 \\
\hline $\begin{array}{l}\text { Pass number of thrombectomy, } \\
\text { median (IQR) }\end{array}$ & -0.12366 & 0.354 & -0.07015 & 0.599 \\
\hline $\begin{array}{l}\text { Total number of operation, } \\
\text { median (IQR) }\end{array}$ & -0.16219 & 0.243 & -0.10225 & 0.466 \\
\hline Safety outcomes & & & & \\
\hline Symptomatic ICH within $24 \mathrm{~h}$ & $0.522(0.244-1.116)$ & 0.093 & $0.453(0.198-1.036)$ & 0.061 \\
\hline Any $\mathrm{ICH}$ within $24 \mathrm{~h}$ & $0.816(0.515-1.29 I)$ & 0.385 & $0.839(0.5 \mid 3-1.374)$ & 0.486 \\
\hline $\mathrm{PH}-2$ within $24 \mathrm{~h}$ & $0.318(0.114-0.890)$ & 0.029 & $0.287(0.096-0.856)$ & 0.025 \\
\hline Death within $90 \mathrm{~d}$ & $0.813(0.484-1.365)$ & 0.433 & $0.775(0.450-1.335)$ & 0.359 \\
\hline Distal or new territorial embolization & $0.403(0.164-0.990)$ & 0.047 & $0.450(0.178-1.141)$ & 0.093 \\
\hline Total complications & $0.463(0.234-0.916)$ & 0.027 & $0.499(0.248-1.004)$ & 0.052 \\
\hline
\end{tabular}

Notes: Model I was adjusted for men, preoperative anticoagulants, aspiration catheter, intraoperative heparin, and the use of IVT. Model 2 was additional adjusted for baseline NIHSS, ASPECTS, location of the occluded vessels, and TOAST classification.

a study examining the factors associated with a favorable functional outcome after MT in elderly patients, pretreatment with IVT was found to be related to a better functional outcome. ${ }^{15}$ In contrast, some studies have revealed no association of IVT pre-treatment prior to MT with prognosis in elderly patients. ${ }^{16-18}$ It is important to note that those studies were not designed to compare the effects of direct MT and bridging MT. Furthermore, some of those studies were not multicenter studies. The present study makes up for these confounders. Given some studies defined elderly patients as $\geq 80$ years old, we performed an exploratory subgroup analysis stratified by age ( $65-79$ vs $\geq 80$ years), but found no difference in the 90-day mRS of patients between these subgroups.

In terms of safety, we found bridging MT increased the incidence of PH-2 and showed a trend toward a higher incidence of $\mathrm{sICH}(10.06 \%$ vs $5.67 \%$, respectively; adjusted $P=0.061)$. The SKIP trial and two multicenter retrospective studies also showed that bridging thrombectomy had a higher risk of $\mathrm{ICH},{ }^{11,14,19}$ which support our result to a certain extent. This suggests that IVT increases the risk of ICH in elderly patients. In fact, some studies have also shown that IVT may be associated with an increased risk of sICH in elderly patients, ${ }^{20,21}$ and that Asian patients may have a higher risk of sICH after IVT. ${ }^{22}$ Therefore, we believe that bridging MT is probably associated with a higher risk of sICH. The relatively small sample size might explain the lack of a statistically significant difference in the incidence of sICH. Further studies with larger cohorts are needed to explore this issue. Furthermore, the bridging MT group showed a trend toward more procedure-related complications, especially distal or new territorial embolization, which is consistent with the DEVT trial. ${ }^{9}$ This may be related to thrombus migration and resolution caused by intravenous alteplase. ${ }^{23}$ Bridging thrombolysis has also been found to be a risk factor for periprocedural 
Table 4 The 90-Day mRS Between Bridging and Direct MT in Patients of Aged 65-79 and $\geq 80$ Years Old

\begin{tabular}{|l|c|c|c|c|c|c|}
\hline & \multicolumn{3}{|c|}{ 65-79 } & \multicolumn{2}{c|}{$\geq 80$} \\
\cline { 2 - 7 } & BMT (N=148) & DMT (N=246) & P-value & BMT (N=39) & DMT (N=49) & P-value \\
\hline mRS 0-1 & $53(35.81)$ & $88(35.77)$ & 0.994 & $14(35.90)$ & $13(26.53)$ & 0.344 \\
mRS 2-6 & $95(64.19)$ & $158(64.23)$ & & $25(64.10)$ & $36(73.47)$ & $15(38.46)$ \\
mRS 0-2 & $56(37.84)$ & $95(38.62)$ & 0.877 & $24(61.54)$ & $31(63.27)$ & 0.868 \\
mRS 3-6 & $92(62.16)$ & $151(61.38)$ & & $18(46.15)$ & $22(44.90)$ & 0.906 \\
mRS 0-3 & $68(45.95)$ & $122(49.59)$ & 0.483 & $21(53.85)$ & $27(55.10)$ & \\
mRS 4-6 & $80(54.05)$ & $124(50.41)$ & & & & \\
\hline
\end{tabular}

thrombus fragmentation and subsequent downstream embolism, ${ }^{24}$ which further supports our conclusion. Although bridging MT was associated with more HT and periprocedural complications, we found no difference in the 90-day $\mathrm{mRS}$ score between the two treatments. This may be related to the finding that there were no differences in the main factors that could affect the prognosis, such as the NIHSS score and ASPECTS, between the two groups.

In the present study, the time from onset to groin puncture was longer in the bridging MT group. Similar findings were reported in the multicenter registry MR CLEAN (Multicenter Randomized Clinical Trial of Endovascular Treatment of Acute Ischemic Stroke), ${ }^{25}$ which supports our conclusion. Because the time from symptom onset to door was similar between the two groups (90 [53-140] vs 90 [48-144] min, respectively; $P=0.583$ ), the difference in the onset-to-puncture time mainly resulted from the door-to-groin puncture time. The time from door to groin puncture did showed a trend toward being longer in the bridging MT group (144 [100-207] vs 109 [76-158] min, respectively; $P=0.060])$. Another multicenter study from China also showed that bridging MT had a longer door-to-groin puncture time, ${ }^{19}$ which supports our conclusion. Overall, these data suggest that bridging MT might delay the time for endovascular treatment. In the DIRECT-MT trial, the median time from hospital admission to groin puncture in the bridging MT group was only 1.5 min longer than that in the direct MT group (85.5 [70-115] vs 84 [67-105] min, respectively), which is markedly less than that in the present study (144 [100-207] vs 109 [76-158] min, respectively). To some extent, this also suggests that some flaws remain in the emergency management of AIS. For example, some patients may not receive MT immediately after IVT, although guidelines recommend that observation after IVT to assess for a clinical response should not be performed. ${ }^{1}$ Furthermore, patients and their relatives are required to be fully informed and provide written consent before IVT in China, which can also somewhat delay the initiation of treatment.

As a nationwide, multicenter prospective registry program, the advantage of the present study is its high external validity. Further, we provide a reference for elderly patients to help with the selection of direct or bridging MT. However, our study has some limitations, and our findings must be interpreted with caution. First, this was not a randomized controlled trial; unknown confounders affecting the results cannot be completely ruled out, although we adjusted for possible confounding factors. Second, patients treated with IVT and recanalized successfully to avoid MT were not enrolled, which may underestimate the efficacy of IVT. Third, because most patients were treated with alteplase, the effects of different thrombolytic drugs were not analyzed. Fourth, the sample size was relatively small. Finally, there are some missing data in this study. Thus, although relevant statistical methods were used to fill in these missing data, there is the potential for bias.

\section{Conclusion}

Our findings suggest that direct MT has similar efficacy to bridging MT in terms of the 90-day functional outcome in elderly patients with LVO, while bridging MT has a longer onset-to-puncture time and increased risk of HT and procedure-related complications.

\section{Data Sharing Statement}

The data that support the findings of this study are available from the corresponding author Zhongrong Miao (zhongrongm@163.com) upon reasonable request. 


\section{Acknowledgments}

We thank all the ANGEL-ACT study groups for providing the data: Zhongrong Miao, MD; Liqiang Gui, MD; Cunfeng Song, MD; Ya Peng, MD; Jin Wu, MD; Shijun Zhao, MD; Junfeng Zhao, MD; Zhiming Zhou, MD; Yongli Li, MD; Ping Jing, MD; Lei Yang, MD; Yajie Liu, MD; Qingshi Zhao, MD; Yan Liu, MD; Xiaoxiang Peng, MD; Qingchun Gao, MD; Zaiyu Guo, MD; Wenhuo Chen, MD; Weirong Li, MD; Xiaojiang Cheng, MD; Yun Xu, MD; Yongqiang Zhang, MD; Guilian Zhang, MD; Yijiu Lu, MD; Xinyu Lu, MD; Dengxiang Wang, MD; Yan Wang, MD; Hao Li, MD; Yang Hua, MD; Deqin Geng, MD; Haicheng Yuan, MD; Hongwei Wang, MD; Haihua Yang, MD; Zengwu Wang, MD; Liping Wei, MD; Xuancong Liufu, MD; Xiangqun Shi, MD; Juntao Li, MD; Wenwu Yang, MD; Wenji Jing, MD; Xiang Yong, MD; Leyuan Wang, MD; Chunlei Li, MD; Yibin Cao, MD; Qingfeng Zhu, MD; Peng Zhang, MD; Xiang Luo, MD; Shengli Chen, MD; WenWu Peng, MD; Lixin Wang, MD; Xue Wen, MD; Shugui Shi, MD; Wanming Wang, MD; Wang Bo, MD; Pu Yuan, MD; Dong Wang, MD; Haitao Guan, MD; Wenbao Liang, MD; Daliang Ma, MD; Long Chen, MD; Yan Xiao, MD; Xiangdong Xie, MD; Zhonghua Shi, MD; Xiangjun Zeng, MD; Fanfan Su, MD; MingZe Chang, MD; Jijun Yin, MD; Hongxia Sun, MD; Chong Li, MD; Yong Bi, MD; Gang Xie, MD; Yuwu Zhao, MD; Chao Wang, MD; Peng Zhang, MD; Xianjun Wang, MD; Dongqun Li, MD; Hui Liang, MD; Zhonglun Chen, MD; Yan Wang, MD; Yu Xin, Wang, MD; Lin Yin, MD; HongKai Qiu, MD; Jun Wei, MD; Yaxuan Sun, MD; Xiaoya Feng, MD; Weihua Wu, MD; Lianbo Gao, MD; Zhibing Ai, MD; Tan Lan, MD; Li Ding, MD; Qilong Liang, MD; Zhimin Wang, MD; Jianwen Yang, MD; Ping Xu, MD; Wei Dong, MD; Quanle Zheng, MD; Zhenyun Zhu, MD; Liyue Zhao, MD; Qingbo Meng, MD; Yuqing Wei, MD; Xianglin Chen, MD; Wei Wang, MD; Dong Sun, MD; Yongxing Yan, MD; Guangxiong Yuan, MD; Yadong Yang, MD; Jianfeng Zhou, MD; Zhi Yang, MD; Zhenzhong Zhang, MD; Ning Guan, MD; Huihong Wang, MD.

We thank Justin Dean and Angela Morben from Liwen Bianji, Edanz Group China (www.liwenbianji.cn/ac), for editing the English text of a draft of the manuscript.

\section{Funding}

This study is supported by grants from the National Key Research and Development Program of China (2016YFC1301500, 2018YFC1312801), the National
Natural Science Foundation of China (81971116), the Shaanxi Provicial Key Research and Development Project of China (2019ZDLSF01-04), and a Postdoctoral Science Foundation (2019M650773).

\section{Disclosure}

The authors report no conflicts of interest in this work.

\section{References}

1. Powers WJ, Rabinstein AA, Ackerson T, et al. Guidelines for the Early Management of Patients With Acute Ischemic Stroke: 2019 Update to the 2018 Guidelines for the Early Management of Acute Ischemic Stroke: a Guideline for Healthcare Professionals From the American Heart Association/American Stroke Association. Stroke. 2019;50(12):e344-e418. doi:10.1161/STR.0000000000000211

2. Kass-Hout T, Kass-Hout O, Mokin M, et al. Is bridging with intravenous thrombolysis of any benefit in endovascular therapy for acute ischemic stroke? World Neurosurg. 2014;82:e453-8. doi:10.1016/j. wneu.2013.01.097

3. Rai AT, Boo S, Buseman C, et al. Intravenous thrombolysis before endovascular therapy for large vessel strokes can lead to significantly higher hospital costs without improving outcomes. J Neurointerv Surg. 2018;10(1):17-21. doi:10.1136/neurintsurg-2016-012830

4. Sallustio F, Koch G, Alemseged F, et al. Effect of mechanical thrombectomy alone or in combination with intravenous thrombolysis for acute ischemic stroke. $J$ Neurol. 2018;265(12):2875-2880. doi:10.1007/s00415-018-9073-7

5. Guimarães Rocha M, Carvalho A, Rodrigues M, et al. Primary thrombectomy versus combined mechanical thrombectomy and intravenous thrombolysis in large vessel occlusion acute ischemic stroke. J Stroke Cerebrovasc Dis. 2019;28(3):627-631. doi:10.1016/j. jstrokecerebrovasdis.2018.11.002

6. Phan K, Dmytriw AA, Lloyd D, et al. Direct endovascular thrombectomy and bridging strategies for acute ischemic stroke: a network meta-analysis. J Neurointerv Surg. 2019;11(5):443-449. doi:10.1136/ neurintsurg-2018-014260

7. Tsivgoulis G, Katsanos AH, Mavridis D, Magoufis G, Arthur A, Alexandrov AV. Mechanical thrombectomy improves functional outcomes independent of pretreatment with intravenous thrombolysis Stroke. 2016;47(6):1661-1664. doi:10.1161/STROKEAHA.116.013097

8. Yang P, Zhang Y, Zhang L, et al. Endovascular thrombectomy with or without intravenous alteplase in acute stroke. $N$ Engl J Med. 2020;382(21):1981-1993. doi:10.1056/NEJMoa2001123

9. Zi W, Qiu Z, Li F, et al. Effect of endovascular treatment alone vs intravenous alteplase plus endovascular treatment on functional independence in patients with acute ischemic stroke: the DEVT Randomized Clinical Trial. JAMA. 2021;325(3):234-243. doi:10.1001/jama.2020.23523

10. Whiteley WN, Slot KB, Fernandes P, Sandercock P, Wardlaw J. Risk factors for intracranial hemorrhage in acute ischemic stroke patients treated with recombinant tissue plasminogen activator: a systematic review and meta-analysis of 55 studies. Stroke. 2012;43 (11):2904-2909. doi:10.1161/STROKEAHA.112.665331

11. Tong X, Wang Y, Fiehler J, et al. Thrombectomy versus combined thrombolysis and thrombectomy in patients with acute stroke: a Matched-Control Study. Stroke. 2021;52:1589-1600. doi:10.1161/ STROKEAHA.120.031599

12. von Kummer R, Broderick JP, Campbell BC, et al. The Heidelberg bleeding classification: classification of bleeding events after ischemic stroke and reperfusion therapy. Stroke. 2015;46 (10):2981-2986. doi:10.1161/STROKEAHA.115.010049 
13. Hacke W, Kaste M, Fieschi C, et al. Randomised double-blind placebo-controlled trial of thrombolytic therapy with intravenous alteplase in acute ischaemic stroke (ECASS II). Second European-Australasian Acute Stroke Study Investigators. Lancet. 1998;352(9136):1245-1251. doi:10.1016/s0140-6736(98)08020-9

14. Suzuki K, Matsumaru Y, Takeuchi M, et al. Effect of mechanical thrombectomy without vs with intravenous thrombolysis on functional outcome among patients with acute ischemic stroke: the SKIP Randomized Clinical Trial. JAMA. 2021;325(3):244-253. doi:10.1001/jama.2020.23522

15. Barral M, Lassalle L, Dargazanli C, et al. Predictors of favorable outcome after mechanical thrombectomy for anterior circulation acute ischemic stroke in octogenarians. J Neuroradiol. 2018;45 (4):211-216. doi:10.1016/j.neurad.2018.01.055

16. Meyer L, Alexandrou M, Flottmann F, et al. Endovascular treatment of very elderly patients aged $\geq 90$ with acute ischemic stroke. $J \mathrm{Am}$ Heart Assoc. 2020;9(5):e014447. doi:10.1161/JAHA.119.014447

17. Alawieh A, Starke RM, Chatterjee AR, et al. Outcomes of endovascular thrombectomy in the elderly: a 'real-world' multicenter study. $J$ Neurointerv Surg. 2019;11(6):545-553. doi:10.1136/neurintsurg2018-014289

18. Meyer L, Alexandrou M, Leischner H, et al. Mechanical thrombectomy in nonagenarians with acute ischemic stroke. J Neurointerv Surg. 2019;11(11):1091-1094. doi:10.1136/neurintsurg-2019-014785

19. Wang H, Zi W, Hao Y, et al. Direct endovascular treatment: an alternative for bridging therapy in anterior circulation large-vessel occlusion stroke. Eur J Neurol. 2017;24(7):935-943. doi:10.1111/ ene. 13311
20. Wardlaw JM, Murray V, Berge E, et al. Recombinant tissue plasminogen activator for acute ischaemic stroke: an updated systematic review and meta-analysis. Lancet. 2012;379(9834):2364-2372. doi:10.1016/S0140-6736(12)60738-7

21. Emberson J, Lees KR, Lyden P, et al. Effect of treatment delay, age, and stroke severity on the effects of intravenous thrombolysis with alteplase for acute ischaemic stroke: a meta-analysis of individual patient data from randomised trials. Lancet. 2014;384 (9958):1929-1935. doi:10.1016/S0140-6736(14)60584-5

22. Menon BK, Saver JL, Prabhakaran S, et al. Risk score for intracranial hemorrhage in patients with acute ischemic stroke treated with intravenous tissue-type plasminogen activator. Stroke. 2012;43 (9):2293-2299. doi:10.1161/STROKEAHA.112.660415

23. Alves HC, Treurniet KM, Jansen IGH, et al. Thrombus migration paradox in patients with acute ischemic stroke. Stroke. 2019;50 (11):3156-3163. doi:10.1161/STROKEAHA.119.026107

24. Kaesmacher J, Boeckh-Behrens T, Simon S, et al. Risk of thrombus fragmentation during endovascular stroke treatment. AJNR Am $J$ Neuroradiol. 2017;38(5):991-998. doi:10.3174/ajnr.A5105

25. Chalos V, LeCouffe NE, Uyttenboogaart M, et al. Endovascular treatment with or without prior intravenous alteplase for acute ischemic stroke. J Am Heart Assoc. 2019;8(11):e011592. doi:10.1161/JAHA.118.011592
Clinical Interventions in Aging

\section{Publish your work in this journal}

Clinical Interventions in Aging is an international, peer-reviewed journal focusing on evidence-based reports on the value or lack thereof of treatments intended to prevent or delay the onset of maladaptive correlates of aging in human beings. This journal is indexed on PubMed Central, MedLine, CAS, Scopus and the Elsevier

\section{Dovepress}

Bibliographic databases. The manuscript management system is completely online and includes a very quick and fair peer-review system, which is all easy to use. Visit http://www.dovepress.com/ testimonials.php to read real quotes from published authors. 